\title{
Model of black hole evolution
}

\author{
J. G. Russo \\ Theory Division, CERN, CH-1211 Geneva 23, Switzerland \\ (Received 22 February 1996; revised manuscript received 16 September 1996)
}

\begin{abstract}
We investigate a model where the quantum dynamics of black hole evaporation is determined by imposing a boundary on the apparent horizon with suitable boundary conditions. An unconventional scenario for the evolution emerges: only an insignificant fraction of energy of order $(m G)^{-1}$ is radiated out; the outgoing wave carries a very small part of the quantum-mechanical information of the collapsed body, the bulk of the information remaining in the final stable black hole geometry. [S0556-2821(97)00202-6]
\end{abstract}

PACS number(s): 04.70.Dy, 04.20.Gz

\section{INTRODUCTION}

It has been argued that, due to backreaction effects, the Hawking model of black hole evaporation [1] may break down long before the evaporation is complete [2]. Because of the exponential redshift, the outgoing modes arise from a reservoir of trans-Planckian energies, with frequencies even higher than the total black hole mass. If a Planck-scale cutoff is imposed before the horizon, it seems that there would be only a scarce amount of outgoing modes, and black holes would lose an insignificant mass by evaporation [3]. Lacking the fundamental short-distance theory, by the time the outgoing modes arise with Planck frequencies, some extra assumption is needed. Extrapolating the Hawking radiation into this region leads to paradoxes, e.g., loss of quantum coherence. However, a concrete alternative scenario to the Hawking model has been elusive so far. problem should be formulated $[4,5]$. The idea is that the description of physics which is appropriate to external observers may require imposing a phenomenological boundary on a surface located about one Planck unit away from the event horizon, where gravitational self-interactions become very strong [4]. In this paper we will attempt a more precise construction. A novel theory of black hole evaporation will be examined, which is based on the assumption that the adequate framework for outside observers is a quantum theory with a boundary on the apparent horizon. The outgoing flux of energy in this model will coincide with the one predicted by the Hawking model only in the region which is not causally connected with the apparent horizon. By that retarded time the Hawking radiation flux is still negligible. In the region in causal contact with the boundary the total flux will be very small and it will exponentially go to zero. As a result, the final state will contain a stable geometry with approximately the same mass as the Arnowitt-Derer-Misner (ADM) mass of the original configuration. Only an energy of order $(m G)^{-1}$ will be evaporated.

A scenario where the Hawking radiation stops leaving a macroscopic black hole was contemplated in [6] as a possible solution of the information problem (for a discussion of its weak points see, e.g., [7]). There it was suggested that the radiation should stop when a certain bound on the information content is saturated. This is not what the present model predicts, but the way the information paradox is resolved is
Recently, there have been some indications on how the

similar, the Hawking process terminates and the information remains stored in the final black hole geometry.

\section{INFORMATION LOSS PROBLEM}

In order for a black hole to be able to evaporate a significant part of its mass, the Hawking model needs to be extrapolated up to retarded times which are causally connected with exponentially small proper distances $\sim \exp \left(-G M^{2}\right)$ from the event horizon. Because of the redshift, an outgoing mode of average frequency $\omega \sim(M G)^{-1}$ would have a frequency $(M G)^{-1} \exp \left(G M^{2}\right)$ at the moment it arises from the horizon, which physically seems unacceptable (in particular, for $M^{2} \gg 1 / G$, higher than the black hole mass), so backreaction effects should be important. The critical time at which corrections to the Hawking model may be expected is when a distant observer enters in causal contact with a surface located about one Planck unit of proper distance away from the event horizon. (This is called the stretched horizon in $[8,4]$; for a discussion of the different regions which are relevant to the evaporation problem, see [9].) The outgoing modes arising from this surface have Planck-scale frequencies, i.e., of order $1 / \sqrt{G}$. Clearly, lacking knowledge of Planck-scale physics, some assumptions are needed in order to calculate the radiation beyond this point. This is a crucial step, since until this time only an energy of order $(G M)^{-1}$ has been evaporated. The justifications underlying the Hawking theory are more or less the following. ${ }^{1}$ For large black holes the curvature is very small at the horizon (i.e., much less than Planck-scale curvatures, $R \ll G^{-1}$ ), so no quantum gravity effects should be expected in this region; the appearance of an outgoing mode is a global effect and it is meaningless to assign it a frequency $\sim(G M)^{-1} \exp \left(G M^{2}\right)$, an inertial observer falling into the black hole sees no strong quantum gravity effects at the moment it crosses the horizon. This picture leads however to several problems. The most serious one is probably loss of quantum coherence: if the initial state was a pure state, the final state, after the black hole disappearance, will be a mixed state and will contain no quantum-mechanical information of the wave function of the collapsing object. A sec-

\footnotetext{
${ }^{1}$ For a general review on the information loss problem see, e.g., [7].
} 
ond problem is that at the end point of the evaporation a naked singularity remains exposed to outside observers. In all dynamical situations that have been studied singularities have always managed to remain hidden behind a horizon. Accepting naked singularities leads to a number of problems, in particular, to a conflict with predictability [10]. Either one is to give up quantum mechanics (and cosmic censorship hypothesis) or else revisite the assumptions of ignoring the problem of Planck frequencies.

There is a loophole in the above reasoning which has led to 't Hooft to formulate the problem in another way. Indeed, the inertial infalling observer cannot be used to argue that outgoing radiation at sub-Planckian distances from the horizon is not affected by quantum gravity effects, since this observer sees no Hawking radiation at all. The speculation of 't Hooft and Susskind is that the quantum gravity effects in this region should be such that radiation still exists but it is no longer exactly thermal; it should contain all the original quantum-mechanical information encoded in subtle correlations, so that unitarity of the evolution is preserved. This interesting approach nevertheless has some problems.

(1) There is no concrete model implementing this picture, despite some attempts. In particular, there are no testable predictions. For the time being it remains a speculation.

(2) There is no unique evolution of the information: for the out observer the information is absorbed by the horizon and then reemitted; for an ingoing observer the information goes into the black hole undisturbed. In this picture the horizon acts as a "quantum xerox," duplicating the quantummechanical information.

(3) As in the Hawking model, one has to accept naked singularities. By the end of the evaporation, Planckian curvatures are exposed to the outside world.

In order to understand what other kind of physics could be expected in this conflictive region, now we explore another possible assumption. The basic idea is that the out observer does not need to know all the details of the physics inside the black hole up to the singularity in order to describe the out physics. One can imagine that the correct quantum theory for external observers requires a boundary at a suitable place. There is a natural place where a boundary can be imposed, namely the apparent horizon, which (for spherically symmetric configurations) simply amounts to exclude the region where the contours $r=$ const are spacelike (see, e.g., [9]). This would mean that there would be a change of the out physics in the region causally connected with the apparent horizon. This is precisely what is desired, since the radiation received in this region originates from about one Planck unit of proper distance away from the horizon, so it as well separates the Planck physics zone. Reflecting boundary conditions can be straightforwardly imposed when the apparent horizon is timelike. The resulting formulas can then be easily continued to the regime where the apparent horizon is spacelike by a continuous deformation of the apparent horizon curve. The predictions of this model is that the Hawking radiation essentially stops in the region causally connected with the trapped surface. The final state is a stable black hole plus minor emission carrying a total energy of order $(m G)^{-1}$. It resolves the problem of information loss and the singularity is never exposed to outside observers. It also avoids loss of quantum coherence with no need of invoking "duplication of information;", here there is a logical evolu- tion of the quantum-mechanical information. As we shall see, in the spherical symmetric case everything is calculable to leading order in $M_{P}^{2} / m^{2}$. In particular, the total emitted energy can be very accurately predicted in terms of the details of the incoming energy. There are a priori two problems: (1) The apparent horizon as a boundary is put by hand. A further justification would be desirable. (2) The physics of the external world is described $a$ s if there was a wall at the apparent horizon. This is in contrast with the idea that nothing so drastic should happen to a free-falling object when encountering the apparent horizon.

Concerning the first point, there is a justification in $1+1$ dimensions: the boundary at the apparent horizon follows automatically if one assumes that an $S$ matrix exists and that it describes the usual semiclassical theory below the threshold for black hole formation [11]. Indeed, in the $S$-matrix formalism, the physics above thresholds is governed by analytic continuation. In $1+1$ dimensions, it can be shown that the supercritical regime which is obtained from the subcritical regime by analytic continuation is mathematically equivalent to the black hole theory with a boundary at the apparent horizon [11]. (In 3+1 dimensions, the dynamics is more complicated, because the $S$-matrix may also be defined by analytic continuation in the transverse variables such as the impact parameter or its conjugate, the momentum transfer $t$.) In any case, it would be more satisfactory to have a derivation of this boundary from first principles, which presently, lacking a fundamental short-distance theory, seems an untractable problem. Why should the correct formalism exclude the region where the contours $r=$ const are spacelike? What would be the nature of Planck-scale physics which could justify the boundary? Here trans-Planckian modes are suppressed, implying an ultraviolet softening at the Planck scale, which is consistent with what is generally expected in quantum gravity. Indeed, the boundary has essentially the same effect as imposing a cutoff at a Planckscale frequency, which leads to a termination of the Hawking process [3].

Concerning the second point, we recall that an outside observer reaches infinite time before the collapsing macroscopic matter reaches the apparent horizon, so for him the question has no physical significance. The question is whether, to an inertial ingoing observer, free-falling matter will pass undisturbed through the apparent horizon. The description of physics in the infalling frame, where there is no Hawking radiation at all, clearly has to be different. For sufficiently large black holes, curvature is very small at the horizon, so in this region physics should be described by the ordinary Einstein equations. We will adopt the viewpoint that the "barrier" at the apparent horizon is just an artifact of the out description. Both descriptions should be complementary in the sense of quantum mechanics. The notion of complementarity in this picture is in fact weaker than in $[2,4]$, where one needs to assume that the information of the objects going inside the black hole must also come out in the Hawking radiation. Indeed, while as in $[2,4]$ we need to assume that outgoing modes, before leaving the horizon region, undergo strong gravitational effects, in those works these effects must "destroy" the infalling object, extract its quantum-mechanical information, and transfer it to the outgoing modes, even before this reaches the apparent horizon. 
Here "complementarity" just means that the quantum-field theoretical description which is appropriate to an outside observer does not need to be extrapolated up to the singularity; instead, simple Dirichlet-, or Neumann-type boundary conditions should be imposed to the quantum fields at the apparent horizon.

\section{DETERMINATION OF THE APPARENT HORIZON FOR GENERAL COLLAPSING MATTER}

Let us restrict our attention to spherically symmetric configurations:

$$
d s^{2}=g_{i j}\left(x^{0}, x^{1}\right) d x^{i} d x^{j}+r^{2}\left(x^{0}, x^{1}\right) d \Omega^{2}, \quad i, j=0,1 .
$$

In this spherically symmetric space-time, the location of the apparent horizon is determined by $g^{i j} \partial_{i} r \partial_{j} r=0$ (see, e.g., [9]). In the conformal gauge, $g_{i j}\left(x^{0}, x^{1}\right) d x^{i} d x^{j}$ $=e^{2 \rho(U, V)} d U d V$, this equation takes the form $\partial_{U} r \partial_{V} r=0$. For the part of $U, V$ space which is physically relevant in the process of gravitational collapse, the apparent horizon will be simply given by the equation $\partial_{V} r=0$.

Let $r=r(U, V), \rho=\rho(U, V)$ be the classical solution of the Einstein equations for a given infalling spherically symmetric configuration $T_{\mu \nu}$. For simplicity only massless matter will be considered. In the conformal gauge, the classical Einstein equations for the $g_{U U}$ and $g_{V V}$ components are given by

$$
\begin{gathered}
\partial_{U}^{2} r-2 \partial_{U} \rho \partial_{U} r=-4 \pi G r T_{U U}, \\
\partial_{V}^{2} r-2 \partial_{V} \rho \partial_{V} r=-4 \pi G r T_{V V},
\end{gathered}
$$

where $T_{V V}$ and $T_{U U}$ represent incoming and outgoing energy-density fluxes. Let the apparent horizon curve be classically given by

$$
U=-P(V) \text {. }
$$

It is easy to obtain $r(U, V)$ in the neighborhood of the apparent horizon. Expanding around $U=-P(V)$, we have

$$
\partial_{V} r^{2} \equiv f(U, V)=-F(V)[U+P(V)]+O\left([U+P(V)]^{2}\right) .
$$

By a conformal transformation one can always set $F(V) d V \rightarrow$ const $d V$, so that the equation simply becomes

$$
\partial_{V} r^{2}=- \text { const } \times[U+P(V)]+\cdots
$$

It is convenient to choose the multiplicative constant equal to $2 e^{-1}$ [cf. Eqs. (3.6) and (3.8)]. By integration we obtain

$$
\begin{aligned}
r^{2}(U, V)= & (2 M(V) G)^{2}-2 e^{-1} V[U+P(V)] \\
& +O\left([U+P(V)]^{2}\right),
\end{aligned}
$$

where a possible additive function $f(U)=c[U$ $+P(V)]+\cdots$ is removed by a shift of $V$, and a function $M(V)$ was introduced, defined by

$$
2 e G^{2} \frac{d M^{2}(V)}{d V}=V \frac{d P(V)}{d V} .
$$

Using Eqs. (3.2), (3.3), and (3.4), the functions $M(V)$ and $P(V)$ can be related to the incoming energy-momentum tensor. In particular, evaluating the $V V$ constraint (3.2) near the apparent horizon, the second term can be dropped, and one finds

$$
\frac{d P(V)}{d V} \cong \frac{T_{V V}}{\mathcal{T}(V)}, \quad \mathcal{T}(V) \equiv\left[16 \pi e G^{3} M^{2}(V)\right]^{-1}
$$

To fix the notation, let us consider the static Schwarzschild geometry. The standard connection with Kruskal coordinates, $U, V$ is given by

$$
2 m G(r-2 m G) e^{r / 2 m G}=-V(U+p), \quad p=2 m G,
$$

$$
\begin{aligned}
& U+p=-2 m G e^{-u / 4 m G}, \quad V=2 m G e^{v / 4 m G}, \\
& v, u=t \pm r^{*}, \quad r^{*}=r+2 m G \ln (r-2 m G) .
\end{aligned}
$$

In this case the apparent horizon coincides with the event horizon. The solution of $\partial_{V} r=0$ is $U=-p$. Expanding $r(U, V)$ in Eq. (3.6) near $U=-p$ one obtains

$$
r^{2} \cong(2 m G)^{2}-2 e^{-1} V(U+p)+O\left((U+p)^{2}\right) .
$$

For a dynamically formed black hole, assuming that $T_{V V}$ vanishes for $V>V_{1}, m=M\left(V_{1}\right)$ will represent the total $\mathrm{ADM}$ mass of the collapsing body, and $p=P\left(V_{1}\right)$ will be associated with the total infalling Kruskal momentum.

The equation of the apparent horizon in the absence of incoming fluxes was determined in [9]. It is easy to generalize this calculation to incorporate infalling matter. The equation

$$
\frac{\partial r(U, V)}{\partial V}=0
$$

can be written in terms of the total derivative on the apparent horizon curve, $r=r_{\mathrm{AH}}(U, V(U))$,

$$
0=\frac{d r_{\mathrm{AH}}}{d U}-\frac{\partial r_{\mathrm{AH}}}{\partial U} \cong \frac{d r_{\mathrm{AH}}}{d U}+\frac{1}{2 e M G} V .
$$

For a large Schwarzschild black hole, $r_{\mathrm{AH}} \cong 2 M G$, so that

$$
-\frac{V}{2 e M G}=\frac{d r_{\mathrm{AH}}}{d U} \cong 2 G \frac{d M}{d U} .
$$

Let us determine $d M / d U$. In the vicinity of the horizon, the mass loss is dictated by the Stefan-Boltzmann law, and the mass given is in terms of the incoming energy-density flux,

$$
\frac{d M}{d v}=\left(-N \frac{\pi^{2}}{30} T_{H}^{4}+T_{v v}\right)\left(4 \pi r_{s}^{2}\right), \quad r_{s}=2 M(V) G
$$

where $N$ represents the number of scalar field degrees of freedom (as shown below, in this model the mass loss will be compensated with an extra negative-energy contribution coming from the lower branch of the apparent horizon; an external observer can only measure the sum of two fluxes). Using 


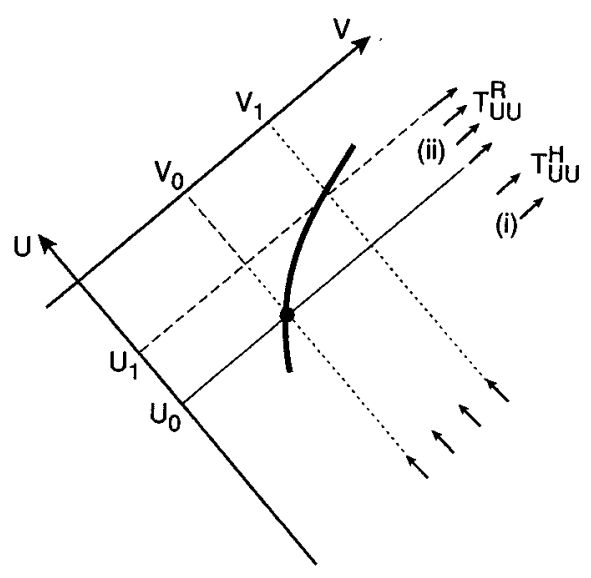

FIG. 1. Apparent horizon for an incident flux less than critical.

$$
\frac{d M}{d U}=\frac{d M}{d v} \frac{d v}{d V} \frac{d V(U)}{d U}
$$

and Eqs. (3.7), (3.9), and (3.10), one obtains

$$
\frac{d V}{d U}\left[-\frac{N e G m^{2}}{480 \pi M^{2} V^{2}}+\frac{T_{V V}}{\mathcal{T}(V)}\right]=-1 .
$$

From Eq. (3.11) [see also Eq. (3.10)] we see that there is a critical value of the incident energy-density flux for which $d V / d U$ changes sign: for lower $T_{V V}$ the apparent horizon is time-like; for larger $T_{V V}$, it is spacelike. Note that a spacelike apparent horizon necessarily involves a black hole geometry, since it implies that the curve $r(U, V)=0$ is spacelike. In Minkowski coordinates:

$$
\left.T_{v v}^{\mathrm{cr}}\right|_{\mathrm{AH}}=N \frac{\pi^{2}}{30} T_{H}^{4}=\frac{N}{122880 \pi^{2} G^{4} M^{4}} .
$$

Equation (3.11) can be easily integrated when $V$ is close to $V_{1}$, where $M(V) \cong m$. In this region the apparent horizon curve takes the simple form

$$
V[U+P(V)] \cong-k G, \quad k=\frac{N e}{480 \pi} .
$$

\section{BOUNDARY CONDITIONS AT THE APPARENT HORIZON}

Following the discussion of Sec. II, now we would like to explore the consequences of postulating a boundary at the apparent horizon. Let us first discuss a situation where the incoming energy density flux is less than $T_{v v}^{\mathrm{cr}}$ in the vicinity of the apparent horizon, so that it is timelike (see Fig. 1). It may be assumed that this subcritical incident flux is striking on the apparent horizon of an already formed black hole. The simplest boundary condition is that this low energy-density matter is just reflected on a timelike apparent horizon. This subcritical reflection must be interpreted as a quantum effect, since $T_{v v}^{\mathrm{cr}}$ vanishes in the classical limit.

In the classical theory, a reflection on a boundary $V(U)$ is a relation of the form

$$
T_{U U}=T_{V V}\left(\frac{d V}{d U}\right)^{2}
$$

Let us now consider the quantum theory. Define the "in" vacuum $|0\rangle_{\text {in }}$ as being the state annihilated by the negative frequency modes with respect to the "in" advanced and retarded times $t \pm r$, and similarly for $|0\rangle_{\text {out }}$, in terms of the " 'out' advanced and retarded times, $v, u=t \pm r^{*}$. Let $\hat{T}_{U U}$ be the energy-momentum tensor operator which is normal ordered with respect to the "out" vacuum, i.e., ${ }_{\text {out }}\left\langle 0\left|\hat{T}_{U U}\right| 0\right\rangle_{\text {out }}=0$, and let $\hat{T}_{V V}$ the operator associated with incoming (advanced) energy-momentum flux from $\mathcal{J}^{-}$, which is normal ordered with respect to the "in" vacuum, ${ }_{\text {in }}\left\langle 0\left|\hat{T}_{V V}\right| 0\right\rangle_{\text {in }}=0$. Any two composite operators $\hat{T}_{U U}$ and $\hat{T}_{U U}^{\prime}$ differing only in the normal-ordering subtraction, will be related in the form, $\hat{T}_{U U}^{\prime}=\hat{T}_{U U}-\hat{t}_{U U}$, where, for free fields, $\hat{t}_{U U}$ will be a $c$ number. Let us introduce the shorthand notation $T_{U U}={ }_{\text {in }}\left\langle 0\left|\hat{T}_{U U}\right| 0\right\rangle_{\text {in }}$ and $T_{V V}={ }_{\text {in }}\left\langle\alpha\left|\hat{T}_{V V}\right| \alpha\right\rangle_{\text {in }}$ where $|\alpha\rangle_{\text {in }}$ is the quantum state representing the collapsing matter. Thus, in the quantum theory the reflection condition (4.1) can be generically written in the form

$$
T_{U U}-t_{U U}=\left(\frac{d V}{d U}\right)^{2}\left(T_{V V}-t_{V V}\right)
$$

Because of normal ordering, the operators $\hat{T}_{U U}$ and $\hat{T}_{V V}$ no longer transform as tensors, but their transformation laws typically contain an anomalous piece (see, e.g., [12]). The role of $t_{U U}, t_{V V}$ in Eq. (4.2) is to preserve the tensor transformation properties of the whole term $\hat{\mathcal{T}}_{U U}=\hat{T}_{U U}-\hat{t}_{U U}$. However, this requirement does not uniquely define them, since one could still add to $\hat{\mathcal{T}}_{U U}$ anything that transforms as a tensor. This ambiguity is removed by demanding the physical condition that $T_{U U}=0$ if $|\alpha\rangle_{\text {in }}=|0\rangle_{\text {in }}$. In fact, $t_{U U}$ depends on a certain property of the incoming state $|\alpha\rangle_{\text {in }}$, namely what in Eq. (3.8) was called $p$, representing the total incoming Kruskal momentum. This is because the "out" time, which is used to define the normal ordering of $\hat{T}_{U U}$, depends itself on $p$ [see Eq. (3.7)].

\section{A. $(1+1)$-dimensional model}

Although the calculation given here will not depend on the explicit form of $t_{U U}, t_{V V}$, it is interesting to compare the reflection condition (4.2) with the analogue equation in the $(1+1)$-dimensional model [13], where this relation automatically arises by demanding that the curvature is finite at the boundary, and $t_{U U}, t_{V V}$ are given by simple expressions. In Kruskal coordinates, one finds Eq. (4.2) with

$$
t_{U U}=\frac{\kappa}{(U+p)^{2}}, \quad t_{V V}=\frac{\kappa}{V^{2}}, \quad \kappa=\frac{N}{48},
$$

where the equation of the apparent horizon is given by

$$
V[U+P(V)]=-\kappa,
$$

which is the precise analog of Eq. (3.13). In particular, using Eqs. 4.2-(4.4), in the region (i), where there is no flux impinging on the boundary, one finds 


$$
T_{U U}^{H}=t_{U U}-t_{V V}\left(\frac{d V}{d U}\right)^{2}=\frac{\kappa}{(U+p)^{2}}-\frac{\kappa}{U^{2}},
$$

where we have used the notation $T_{U U}^{H}$ to indicate that, since in region (i) there is nothing to be reflected, this flux must be interpreted as a Hawking radiation flux. Indeed, for $p \gg 0$ (as will be clear later, this is always the case for macroscopic black holes), the second term can be ignored, and one simply has

$$
T_{U U}^{H} \cong \frac{\kappa}{(U+p)^{2}},
$$

which is the usual result that one would derive by the standard calculation of [1].

\section{B. $3+1$ dimensions}

Using Eq. (4.2), in regions (i) and (ii) we thus have the fluxes

$$
\begin{gathered}
T_{U U}^{R}=t_{U U}+\left(\frac{d V}{d U}\right)^{2}\left(T_{V V}-t_{V V}\right), \\
T_{U U}^{H}=t_{U U}-\left(\frac{d V}{d U}\right)^{2} t_{V V} .
\end{gathered}
$$

It is interesting to note that the radiation flux $T_{U U}^{H}$ obtained in this way is consistent with what one would derive from the constraint equations (3.2), upon the replacement of $T_{U U}$ by $T_{U U}-t_{U U}$

$$
T_{U U}^{H}=-(4 \pi G r)^{-1}\left(\partial_{U}^{2} r-2 \partial_{U} \rho \partial_{U} r\right)+t_{U U}, \quad U<U_{0} .
$$

For $V \rightarrow \infty$ the solution in region (i) approaches the classical Schwarzschild solution, so that the first term in Eq. (4.9) vanishes, and one obtains

$$
T_{U U}^{H} \cong t_{U U}, \quad U<U_{0} .
$$

Just as in the $(1+1)$-dimensional model, the second term in Eq. (4.8) is a small correction to Eq. (4.10), which can be neglected near a black hole horizon [the reason is that $t_{U U}$ goes like $1 /(U+p)^{2}$ and it blows up near $U \cong-p$; see Eq. (4.6) and Eq. (5.2) below].

The total energies radiated in regions (i) and (ii) will be given by [we use $4 m G d U=-(U+p) d u$ ]:

$$
\begin{gathered}
E_{\text {out }}^{(\mathrm{i})}=4 \pi \int_{-\infty}^{u_{0}} d u r^{2} T_{u u}=-\frac{\pi}{m G} \int_{-\infty}^{U_{0}} d U(U+p) r^{2} T_{U U}^{H} \\
E_{\mathrm{out}}^{(\mathrm{ii})}=-\frac{\pi}{m G} \int_{U_{0}}^{U_{1}} d U(U+p) r^{2} T_{U U}^{R}
\end{gathered}
$$

Let us now gradually increase $T_{v v}$ above $T_{v v}^{\mathrm{cr}}$ so that a part of the apparent horizon becomes spacelike, as in Fig. 2 [the change of character from timelike to spacelike at $T_{v v}^{\mathrm{cr}}$ is dictated by Eq. (3.11)]. In this process a part of region (i) ends up superposing with region (ii), giving rise to the region (b) of Fig. 2. In this region the two contributions $T_{U U}^{R}$ and

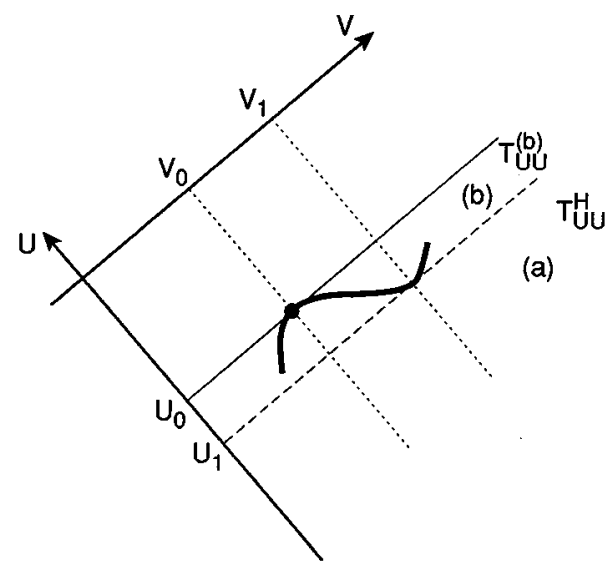

FIG. 2. Apparent horizon for an incident flux greater than critical.

$T_{U U}^{H}$ are thus superposed. The correct outgoing $T_{U U}^{(b)}$ can be obtained by carefully continuing the previous formulas. Now $U_{0}>U_{1}$, so that

$$
\begin{aligned}
\int_{-\infty}^{U_{0}} d U(U+p) T_{U U}^{H}= & \int_{-\infty}^{U_{1}} d U(U+p) T_{U U}^{H} \\
& +\int_{U_{1}}^{U_{0}} d U(U+p) T_{U U}^{H}, \\
\int_{U_{0}}^{U_{1}} d U(U+p) T_{U U}^{R}= & \int_{U_{1}}^{U_{0}} d U(U+p)\left(-T_{U U}^{R}\right) .
\end{aligned}
$$

Therefore, the total energy radiated between $U_{1}$ and $U_{0}$ is

$$
E_{\mathrm{out}}^{(b)}=-\frac{\pi}{m G} \int_{U_{1}}^{U_{0}} d U(U+p) r^{2} T_{U U}^{(b)},
$$

where

$$
T_{U U}^{(b)}=T_{U U}^{H}-T_{U U}^{R}=-T_{V V}\left(\frac{d V}{d U}\right)^{2}
$$

Thus when the apparent horizon is spacelike $T_{U U}^{R}$ contributes with the reverse sign. An extra contribution in region $(b)$ is not a surprise, since the geometry in region $(b)$ is expected to undergo some modification, being in causal contact with the boundary line. The flip of sign can be physically understood as follows. For each given $U^{\prime}$, the geometry at $V^{\prime}$ is determined in terms of the energy that has crossed $U^{\prime}$ at earlier $V<V^{\prime}$. In the presence of the reflecting spacelike wall at $U>U_{1}$, the energy-momentum flux crossing $U_{1}$ cannot be felt by the geometry in region $(b)$. The net effect is that the geometry in region $(b)$ is changed in such a way that the flux $T_{U U}^{R}$ must be subtracted from the outgoing flux. This interpretation is confirmed in [11] for the solvable twodimensional model of [13], where the exact time-dependent geometry, including the geometry in region $(b)$, can be obtained.

It should be noted that only subcritical matter reflects off the (timelike) apparent horizon. The critical energy density (3.12) at the horizon of a massive black hole is extremely 


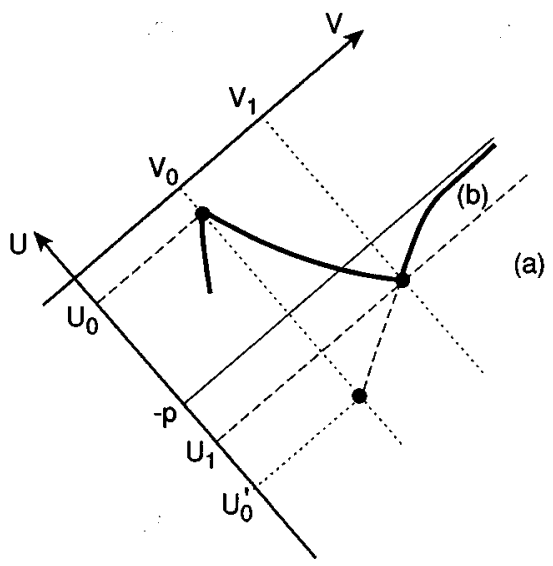

FIG. 3. A macroscopic black hole geometry. The thick line represents the apparent horizon.

low (e.g., $10^{-64} \mathrm{~g} / \mathrm{cm}^{3}$, for a solar mass black hole). For infalling objects with energy density greater than critical, the apparent horizon will be spacelike, and they will just go inside the black hole increasing its mass. As shown below, only a very minor part of their energy and of their information will be emitted.

\section{EVOLUTION OF MACROSCOPIC BLACK HOLES}

Let us consider the evolution of a macroscopic black hole geometry, i.e., with total mass $m \gg m_{P}, m_{P}=1 / \sqrt{G}$. For convenience we will assume that $T_{V V}$ vanishes for $V<V_{0}$ and $V>V_{1}$. For $V>V_{1}$ the geometry is approximately static and given by the Schwarzschild geometry with $m=M\left(V_{1}\right)$ $\gg m_{P}, p=P\left(V_{1}\right) \gg\left|U_{0}\right|$. For $V>V_{1}$ the apparent horizon curve will be given by the equation $V(U+p) \cong-k G$. The geometry is displayed in Fig. 3. As shown in the previous section, the energy-density flux in region $(b)$ is given by [see Eq. (4.14)]

$$
T_{U U}^{(b)}=-T_{V V}\left(\frac{d V}{d U}\right)^{2}
$$

We notice that this outgoing energy-density flux is negative. It will soon be clear that the amount of negative energy radiated in region $(b)$ is a tiny Planck-scale quantity. (In quantum theory the energy density is not positive definite, and global energy positivity will not be violated. It is the tail of the outgoing wave that carries off this bit of negative energy.) What has happened is that the usual Hawking contribution (4.10) has cancelled out in Eq. (4.14) because of the superposition of the two fluxes. The leftover is an insignificant flux, representing a small tail of the wave function coming out in region $(a)$.

First, let us estimate the total (positive) energy radiated in region $(a)$. As is well known, the Hawking radiation flux is significant only near $U=-p$ where it has the form (see, e.g., [12])

$$
T_{u u}^{H} \sim \frac{1}{(G m r)^{2}} \quad \text { or } \quad T_{U U}^{H} \sim \frac{1}{r^{2}(U+p)^{2}}
$$

and it can be neglected for $U<U_{0}^{\prime} \equiv-p-k G / V_{0}$. Thus

$$
\begin{aligned}
E_{\mathrm{out}}^{(a)} & \cong-\frac{\pi}{m G} \int_{U_{0}^{\prime}}^{U_{1}} d U(U+p) r^{2} T_{U U}^{H} \\
& \cong-\frac{k}{4 e m G} \ln \frac{U_{1}+p}{U_{0}^{\prime}+p}=\frac{k}{4 e m G} \ln \frac{V_{1}}{V_{0}},
\end{aligned}
$$

which, indeed, is a small amount of energy. This can be more explicitly seen by relating $\ln \left(V_{1} / V_{0}\right)$ to the physical parameters characterizing the incoming energy-density flux, such as the total energy $m$. In particular, consider an approximately constant ( $v$-independent) flux $T_{v v}$, which is such that $T_{v v} \cong \mathscr{E}$ at $r \sim 2 m G$. The total mass will be given by

$$
m \cong 4 \pi r_{s}^{2} \mathscr{E}\left(v_{1}-v_{0}\right) \sim(m G)^{3} \mathscr{E} \ln \frac{V_{1}}{V_{0}} .
$$

Thus we find

$$
E_{\mathrm{out}}^{(a)} \cong \frac{k}{16}(m G)^{-1} \frac{\mathscr{E}_{\mathrm{cr}}}{\mathscr{E}}, \quad \mathscr{E}_{\mathrm{cr}} \equiv\left(16 \pi e G^{3} m^{2}\right)^{-1}=\mathcal{T}\left(V_{1}\right)
$$

The parameter $\mathscr{E}_{\text {cr }}$ is roughly equal to the critical density at which a uniform spherical body would lie within its Schwarzschild radius [note that $T_{v v}^{\mathrm{cr}}$ is much smaller than $\left.\mathscr{E}_{\mathrm{cr}}, T_{v v}^{\mathrm{cr}} \sim \mathscr{E}_{\mathrm{cr}}\left(m_{p}{ }^{2} / m^{2}\right)\right]$.

Next, we calculate the (negative) energy received in region $(b)$. Let $\left(V_{2}, U_{2} \equiv-p\right)$ be the point at the intersection between the apparent horizon and the null line $U=-p$, i.e., $V_{2}\left[-p+P\left(V_{2}\right)\right]=-k G$. Let us note that for $m \gg m_{P}, V_{2}$ and $V_{1}$ differ by a small quantity (it should be remembered that the splitting between the timelike part of the apparent horizon and the horizon $U=-p$ is a quantum effect). In particular, for a constant density flux one has

$$
\frac{V_{2}}{V_{1}} \cong 1-\frac{k \mathscr{E}_{\mathrm{cr}}}{16 G m^{2} \mathscr{E}}
$$

where we used

$$
-p+P\left(V_{2}\right) \cong(4 m G)^{2} \frac{\mathscr{E}}{\mathscr{E}_{\mathrm{cr}}}\left(V_{1}^{-1}-V_{2}^{-1}\right),
$$

as follows from integrating Eq. (3.5). The outgoing energy momentum tensor in region $(b)$ is given by Eq. (5.1). Since we are only interested in the leading order in $m_{P} / m$, we can use $d U / d V \cong-P^{\prime}(V)$. Inserting Eq. (3.5) into Eq. (5.1), one obtains

$$
T_{U U}^{(b)} \cong \mathcal{T}(V) \frac{d V}{d U}=-\frac{\mathcal{T}^{2}(V)}{T_{V V}} .
$$

$T_{U U}^{(b)}$ carries out information about the small fraction of the infalling matter that arrived at the apparent horizon between $V_{2}$ and $V_{1}$. In Minkowski coordinates,

$$
T_{u u}^{(b)} \cong-\frac{V^{2} \mathcal{T}^{2}(V)}{(8 m G)^{2} T_{v v}} \exp \left(-\frac{u}{2 m G}\right) .
$$

Hence $E_{\mathrm{out}}^{(b)} \cong 4 \pi r_{s}^{2} \Delta u T_{u u}^{(b)}\left(u_{1}\right), \Delta u \sim 2 m G$, 


$$
\begin{gathered}
E_{\mathrm{out}}^{(b)} \cong-\frac{\pi \mathscr{E}_{\mathrm{cr}}^{2}}{2 \mathscr{E}} m G V_{1}^{2} e^{-u_{1} / 2 m G} \cong-a\left(G^{2} m^{3}\right)^{-1}, \\
a=\frac{k^{2} \mathscr{E}_{\mathrm{cr}}}{128 e \mathscr{E}}<1,
\end{gathered}
$$

where we have used $e^{u_{1} / 4 m G}=2 m V_{1} / k$. Thus the emitted negative energy is smaller than $m_{P}^{4} / m^{3}$ in absolute value. Since $m \gg m_{P}$, this is a tiny energy (e.g., for a solar mass black hole, $E_{\mathrm{out}}^{(b)} \sim-10^{-114} m_{P}$ ). From Eqs. (5.4) and (5.7) one finds

$$
\frac{\left|E_{\text {out }}^{(b)}\right|}{E_{\text {out }}^{(a)}} \cong \frac{m_{P}^{2}}{m^{2}} \ll 1 .
$$

Thus the total radiated energy $E_{\text {out }}^{(a)}+E_{\text {out }}^{(b)}$ is positive and of order $E_{\text {out }}^{(a)} \sim(m G)^{-1}$ [see Eq. (5.4)].

\section{CONCLUSIONS}

To summarize, a simple theory of black hole evolution based on reflecting boundary conditions on the apparent horizon was described. The departure from Hawking theory occurs precisely by the time the outgoing modes arise with Planckian frequencies from the vicinity of the horizon (further discussions on the problem of Planck frequencies can be found in $[14,15])$. The sudden fall of the subsequent outgo- ing flux is caused by a contribution from the expanding trapped surface. The total radiated energy is a small (positive) Planckian quantity. The final configuration is a stable black hole geometry, which has retained most of its mass together with the quantum-mechanical information of the original configuration.

The stability of the final geometry can be understood in different ways. It is known that in order to have zero fluxes at infinity [in the present case, in region $(b)$ ], the gravitational field must be greatly modified near the line $U=-p$. This picture is sometimes referred to as the Boulware vacuum choice, defined in terms of the Schwarzschild Killing vector (here the geometry has settled down to this situation dynamically having started from the Unruh vacuum). Accordingly, the geometry in region $(b)$ will be given by the Schwarzschild metric only at far distances from $U=-p$, viz. for $-V(U+p) \gg \exp \left[-\right.$ const $\left.G m^{2}\right]$. This condition is satisfied in the whole of the accessible part of region $(b)$, where $-V(U+p)>k G$, and therefore the corrections to the Schwarzschild metric will be exponentially small in the allowed space-time.

\section{ACKNOWLEDGMENTS}

The author wishes to thank D. Amati for useful discussions and collaboration in the $(1+1)$-dimensional analogue [11], and E. Verlinde for remarks.
[1] S. Hawking, Commun. Math. Phys. 43, 199 (1975).

[2] G. 't Hooft, Nucl. Phys. B256, 727 (1985); B335, 138 (1990).

[3] T. Jacobson, Phys. Rev. D 44, 1731 (1991).

[4] L. Susskind, L. Thorlacius, and J. Uglum, Phys. Rev. D 48, 3743 (1993).

[5] C. Stephens, G. 't Hooft, and B. F. Whiting, Class. Quantum Grav. 11, 621 (1994).

[6] S. Giddings, Phys. Rev. D 46, 1347 (1992).

[7] D. Page, Can. Gen. Relativity 1, (1993).

[8] K. Thorne, R. Price, and D. MacDonald, Black Holes: The Membrane Paradigm (Yale University Press, New Haven, CT, 1986).

[9] J. G. Russo, Phys. Lett. B 359, 69 (1995).
[10] R. M. Wald, General Relativity (University of Chicago Press, Chicago, 1984).

[11] D. Amati and J. G. Russo, "Black holes by analytic continuation,' Report No. hep-th/9602125 (unpublished).

[12] S. M. Christensen and S. A. Fulling, Phys. Rev. D 5, 2088 (1977); N. Birrell and P. Davis, Quantum Fields in Curved Space (Cambridge University Press, Cambridge, England, 1982).

[13] J. G. Russo, L. Susskind, and L. Thorlacius, Phys. Rev. D 46, 3444 (1992); 47, 533 (1993).

[14] Y. Kiem, H. Verlinde, and E. Verlinde, Phys. Rev. D 52, 7053 (1995).

[15] T. Jacobson, Phys. Rev. D 53, 7082 (1996). 